\title{
Knowledge of Nurses Working in Critical Care Areas Regarding Ventilator Associated Pneumonia Prevention Bundles in a Tertiary Level Cardiac Centre
}

\author{
Sandip Bhandari', Medha Sharma², Gentle S Shrestha ${ }^{3}$
}

\section{Author(s) affiliation}

'Department of Anesthesiology,

Shahid Gangalal National Heart

Centre, Kathmandu, Nepal.

${ }^{2}$ Visible Impact, Kathmandu, Nepal.

${ }^{3}$ Department of Anesthesiology,

Maharajgunj Medical Campus,

Tribhuvan University Teaching

Hospital, Maharajgunj, Institute of

Medicine, Kathmandu, Nepal

\section{Corresponding author}

Sandip Bhandari, MBBS, MD

sandipbhandari@hotmail.com

\section{Submitted}

Jun 21, 2020

\section{Accepted}

Jan 10, 2021

\begin{abstract}
\section{Introduction}

Prevention of ventilator associated pneumonia (VAP) is influenced by the knowledge and practices of nurses. This study was conducted to assess the knowledge of nurses working in critical care areas of a tertiary level cardiac centre regarding VAP prevention bundles.
\end{abstract}

\section{Methods}

In this descriptive study, 57 nurses working in critical care areas were included, they completed a self-administered questionaire containing demographic information followed by 18 multiple response questions. With the respondents choosing one or more correct answers, the percentage of correct answer for each options was obtained. For each questions, the correct percentage for all the options were averaged and expressed as average knowledge percent. The significance of association was tested using chisquare and Fisher exact test, with level of significance established at 95\%. Data analysis was done using SPSS version 21.

\section{Results}

With a mean age of 26.8 years, the majority of the nurses had completed Bachelor's degree (75.4\%). Though 91.3\% nurses had experience of more than a year in critical care, $87.7 \%$ of them had not obtained VAP or infection control training. The mean knowledge score of VAP was $70 \pm 7.5 \%$, and $97.4 \%$ had satisfactory knowledge. There was no association between knowledge scores on prevention of VAP and educational qualification $(p=0.26)$ and years of experience in intensive care unit $(p=0.41)$.

\section{Conclusion}

Almost all of the nurses working in critical care areas had satisfactory knowledge regarding VAP bundle, however, they had lower knowledge on the definition and approach to prevent VAP. This study recommends to incorporate more nurses in training related to infection and VAP prevention.

\section{Keywords}

Critical care area, knowledge, nurses, ventilator-associated pneumonia 


\section{INTRODUCTION}

$\mathrm{M}$ echanical ventilation is an important lifesaving technique, yet it is not free from risks. Mechanically ventilated patients face complications such as acute respiratory distress syndrome, pulmonary edema, pulmonary embolism, lobar atelectasis, etc. Among these, ventilator associated pneumonia (VAP) is not only common but also a preventable event of intensive care unit (ICU) infection. ${ }^{1}$ VAP is attributed to mortality of approximately $10 \%$ but varies considerably in different populations. ${ }^{2}$ VAP also increases the duration of mechanical ventilation, stay in ICU and hospital, use of antimicrobials as well as other costs. ${ }^{3,4}$

VAP is defined as chest radiograph showing new or progressive infiltrates, consolidation, cavitation, or pleural effusion that persist for $>48$ hours with the following 2 out of 3 criteria in place: 2

i. Temperature $>38$ degrees Celsius,

ii. White blood cell counts $>12,000$ cells or $<4,000$ per microliter and

iii.Increase in purulent secretion.

The other definition of VAP uses Clinical Pulmonary Infection Score which includes the culture of an organism and arterial blood gas (ABG) picture which has limitations such as the unavailability of $A B G$ machine and issues related to staining, cultures and growth of organisms from the respiratory secretion. Thus, defining VAP by the clinical, radiological and microbial diagnosis has inter-individual variation, ranging the incidence of VAP from 8.3 to $28 \% .{ }^{5,6}$ VAP prevention bundle varies with studies of various time, institute and country itself. A bundle for the prevention of VAP may include: elevation of head of the bed, oral care with chlorhexidine, subglottic suctioning, daily assessment for extubation and the need for proton-pump inhibitors, use of closed suction systems, and maintaining endotracheal cuff pressure at $25 \mathrm{~cm} \mathrm{H}_{2} \mathrm{O}^{7}$ Along with the above we have tested the knowledge regarding hand hygiene, interruption of sedative drugs daily, performance spontaneous breathing trail daily, etc.

Almost all cardiac surgeries are performed with cardio-pulmonary bypass where patient's lungs are not ventilated, except for few cases which are performed off-pump. All cases, be it off-pump or on-pump, arrive at adult and pediatric surgical ICU intubated after surgery. Both these factors are associated with increased risk of infection. Likewise, the resuscitated patient from Emergency Department or Post Cardiac Catheterization Laboratory following myocardial infarction or other procedures arrive intubated in Medical ICU or coronary care unit. The compounding effect of cardiac pathology, endotracheal intubation and non-ventilated lungs might increase the risk of pneumonia. ${ }^{8}$

It is recognized that the prevention of VAP is primarily the responsibility of critical care team. Critical care area is a multidisciplinary area where patients are treated by the team of physicians, intensivists, anaesthesiologists and nurses. Nurses, who are frontliners providing round the clock service have an important role in preventing VAP by decreasing risk factors, recognizing early symptoms and assisting in diagnosis. ${ }^{9}$ So, the health outcome of an ICU patient is significantly influenced by the knowledge, beliefs, and practices of these nurses. Also, studies has shown that improved knowledge of nurses after educational interventions can be associated with decreased rate of VAP. ${ }^{10}$

With the aforementioned rationale, we assessed the knowledge of nurses working in critical care areas of a tertiary level cardiac centre regarding VAP prevention bundles, and explore the association between the level of knowledge and sociodemographic and training factors.

\section{METHODS}

This is a descriptive cross-sectional study conducted in Shahid Gangalal National Heart Centre, Bansbari, Kathmandu, from April to May 2019 after ethical clearance from the Institutional Review Committee of the centre. Study population were 70 nurses working in critical care areas of the centre. Adhering to this centre's protocol to have three months bed side training for nurses before attending critical patients individually, we have excluded eight such nurses in the study. With non-responding five nurses, the data was collected from 57 respondents out of total 70. After securing written informed consent, the respondents self administered the questionnaire. The questionnaire was reviewed by four intensivists working in different hospitals of Nepal with at least five years of experience in ICU. There were two sections in questionaire. While the first section contained demographic information including age, maximum education level attained, experience in ICU and training regarding VAP and infection control, the second section contained 18 multiple response questions. With the respondents choosing one or more correct answers, the percentage of correct answer for each options was obtained. For each questions, the correct percentage for all the options were averaged and expressed as average knowledge percent. With this, the participant could score $0-100 \%$ on each question. Finally, satisfactory knowledge was defined as average knowledge score of $>60 \%$ and unsatisfactory as $<60 \%$ from study done by Ali NS. ${ }^{11}$ The significance of association was tested using chi-square and Fisher exact test, with level of significance established at 95\%. Data analysis was done using SPSS Statistics version 21. 


\section{RESULTS}

All the respondents were female and their mean age was 26.8 years. Respondents in the age group (20-25) years, (25-30) years and $>30$ years were $18(31.6 \%), 28$ (49.1\%), and 11 (19.3\%) nurses respectively. Most of the respondents have completed their Bachelor's degree, either Post Basic Bachelor in Nursing (22/38.6\%) or Bachelor of sciences in Nursing (21/36.8\%), followed by $13(22.8 \%)$ completing certificate level (PCL) in

Table 1. Knowledge of nurses regarding various components of VAP $(n=57)$ (continued on next page.)

$\begin{array}{cc}\text { Components of knowledge regarding } & \text { Correct } \\ \text { ventilator associated pneumonia prevention bundle } & \begin{array}{c}\text { Average } \\ \text { responses }\end{array} \\ \text { score (\%) }\end{array}$

1. Regarding Ventilator associated pneumonia 44.5

a. Pneumonia that occurs 48-72 hours or thereafter following endotracheal tube characterized by the presence of new or progressive infiltrate, sign of systemic infection, changes in sputum characteristics and detection of causative agents (yes) 55 (96.5)

b. Chest radiography finding showing new/progressive infiltrates, consolidation, cavitation or pleural effusion that persist for 48 hours in an intubated patient with 2 out of 3 of following- increased purulent secretion, raised temperature $>38$ degree and WBC counts $>12000 /<4000 /$ microlitre (yes)

c. Clinical pulmonary infection scores are taken into account clinical, physiological, microbiological, and radiographic evidence to allow a numeric value to predict the presence or absence of VAP (yes)

2. How can we prevent VAP

a. Protocolized approach (yes) 60.5

b. Staff education (yes)

c. Creating structures that facilitates bundle adherence (yes)

d. Regular feedback (yes)

3. Regarding ventilation, to prevent VAP

a. We recommend non invasive ventilation over invasive ventilation (yes)

b. Oral intubation is preferred over Nasal intubation (yes)

27 (47.4)

c. Nasal intubation is preferred over oral intubation (no)

$30(52.6)$

d. Both oral and nasal intubation preferred (no)

$52(91.2)$

$57(100)$

4. Regarding gastric feeding

a. Oro-gastric tube is recommended (yes)

98.8

b. Nasogastric tube is recommended (no)

$56(98.2)$

$56(98.2)$

c. Either of them can be used (no)

$57(100)$

5. Patient positioning

a. Prone positioning improve VAP (no)

$52(91.2)$

b. Semi-recumbinant position 30-45 degree is recommended (yes)

c. Positioning don't effect risk of VAP (no)

$54(94.7)$

d. Semi-recumbinant position 45-60 degree is recommended (no)

47 (82.5)

6. Regarding frequency of circuit change

63.7

a. Change circuit every 48 hours or when clinically indicated (no)

$52(91.2)$

b. Change circuit every 72 hours or when clinically indicated (no)

$27(47.4)$

c. Change circuit for every new patient or when clinically indicated (yes)

$30(52.6)$

7. Regarding endotracheal tube

a. ETT with extra lumen for subglottic secretion decrease risk of VAP (yes)

b. ETT with extra lumen for subglottic secretion increase risk of VAP (no)

$56(98.2)$

c. Do not influence risk of VAP (no)

$56(98.2)$

$58(98.2)$

8. Spontaneous breathing trail (SBT)

a. Assess patient's ability to breath while weaning minimal or no ventilatory support (yes)

b. T-piece trail/CPAP equal to PEEP can be used in spontaneous breathing trail for 30-

120 minutes (yes)

c. SBT do prevent VAP (yes) 
Table 1. Knowledge of nurses regarding various components of VAP $(n=57)$ (contd ...)

\section{Components of knowledge regarding ventilator associated pneumonia prevention bundle}

Correct

responses

n (\%)

Average

score $(\%)$
9. Sedation holiday
a. Define as stopping sedation every morning to make patient awake (yes)
$51(89.5)$
b. It is continued till patient become fully awake and obey command or until
$11(19.3)$
comfortable or agitated and deemed to resumption of sedation (yes)
c. Sedation holiday do not prevent VAP (no)
$0(0)$
10. Early mobilisation
a. Its very difficult with multiple life sustaining drugs, catheters, monitors and
$17(29.8)$ sedative medication (yes)
b. Recommended to prevent VAP (yes)
$38(66.7)$
c. Don't have role to prevent VAP (no)
$57(100)$
d. Deep Vein thrombosis prophylaxis prevent VAP (yes)
$31(54.4)$

36.3

11. Frequency of humidifier change
a. Recommended to change every 48 hours or when clinically indicated (no)
$46(80.7)$
b. Recommended to change every 72 hours or when clinically indicated (no)
$28(49.1)$
c. Recommended to change every week or when clinically indicated (yes)
$16(28.1)$
d. Not recommended to improve VAP (no)
$57(100)$

12. Close circuit system

a. It is recommended over open system (yes)

b. Open circuit system is recommended (no)

$57(100)$

$57(100)$

c. Both are not recommended (no)

57 (100)

d. Both have no benefit (no)

57 (100)

13. Kinetic bed
a. Kinetic bed increase risk of VAP (no)
$55(96.5)$
b. Kinetic bed decrease risk of VAP (no)
$6(10.5)$
c. No influence on VAP (no)
$2(3.5)$

14. Regarding endotracheal tube(ETT)

a. Silver coated ETT prevent VAP (yes)

b. ETT with ultra thin polyurethane cuff prevent VAP (yes)

$53(93)$

c. Regular cuff pressure measurement and maintaining it prevent VAP (yes)

$10(17.5)$

$51(89.5)$

15. Oral and digestive tract

a. Chlorhexidine mouth wash prevent VAP (yes)

b. Selective digestive and oral decontamination prevent VAP (no)

$56(98.2)$

$3(5.3)$

c. Measure of residual gastric volume prevent VAP (no)

d. Use of stress ulcer prophylaxis prevent VAP (yes)

16. Regarding trachoestomy and saline installation during suction

a. Early tracheostomy prevent VAP (no)

$42(73.7)$

b. Saline installation during suctioning prevent VAP (no)

$54(94.7)$

c. No role of tracheostomy and Saline installation during suction to prevent VAP (yes)

$35(61.4)$

17. Probiotics and antibiotics prophylaxis

a. Probiotics are useful to prevent VAP (no)

$10(17.5)$

b. Prophylactic antibiotics prevent VAP (no)

c. Both have no role (yes)

$54(94.7)$

18. Hand hygiene

a. Practice of hand hygiene helps to decrease infection in ICU

b. Use of hand accessories- watch, rings increase risk of infection

c. Regular use of gloves to touch patient and IV or central lines prevent infection 


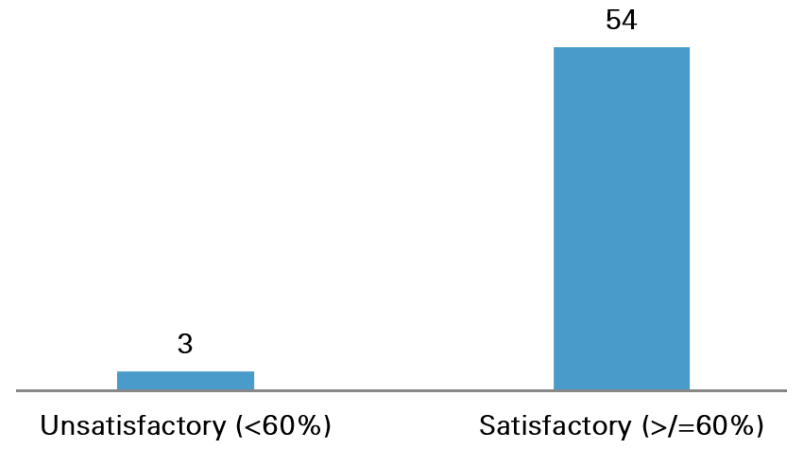

Fig 1. Distribution of knowledge score

nursing, while only one respondent have completed Masters in Nursing. Twenty seven (47.4\%) of respondents had ICU experience of $1-5$ years, while $25(43.9 \%)$ had more than 5 years of experience. Very few respondents had experience of only 3-6 months.

Fifty $(87.7 \%)$ nurses working in critical care areas had not attended VAP or infection control training. Only $7(10.6 \%)$ of them had obtained either of the training and only one respondent had obtained both the training.

The mean average score was $70 \pm 7.5 \%$ (minimum $50.8 \%$, maximum $100 \%$ ) in this study. As shown in figure 1, 54 (94.7\%) respondents had satisfactory knowledge. Only one respondent obtained 100\% score, who happened to be the one attaining both VAP and infection prevention training.

Each component of knowledge regarding VAP prevention bundle was further analyzed as shown in table 1. It was found that all the respondents had knowledge that a close circuit system is recommended over an open system. The respondents had average knowledge of $98.8 \%$ regarding gastric tube feeding. The endotracheal tube (ETT) with an extra lumen for subglottic secretion decreases the risk of VAP is known by $98.2 \%$ of respondent. Approximately 43 (76\%) nurses thought there is no role of tracheostomy and saline instillation during suction to prevent VAP. The respondents had the least knowledge about sedation holiday (69.3\%) and kinetic bed (36.8\%). Eighty percent of respondents did not know that the sedation holiday is continued till the patient becomes fully awake, and comfortable or deemed to resumtion of sedation due to agitation. None knew that sedation holiday prevent VAP. Also, 89.5\% thought that the kinetic bed decreases the risk of VAP.

Association was not significant between the knowledge scores and age $(p=0.21)$, educational qualification $(p=0.26)$, years of experience in ICUs $(p=0.41)$ and training experience $(p=0.63)$ considering p-value significant at 0.05 .

\section{DISCUSSION}

This study revealed that 54 (94.7\%) nurses working in critical care areas had satisfactory knowledge scores about VAP irrespective of their work experience in critical care area and education level. The proper definition of VAP is known to 18 (31.6\%) nurses. Majority of the nurses acquired knowledge about critical patients' care from their seniors working in ICU as many nursing curriculum do not prioritize sessions on critical care. This study also depicts overall VAP knowledge score of $70 \pm 7.5 \%$ among those nurses which might be attributed to their experience of more than a year in a critical area $(91.3 \%)$ rather than their education level as almost all of them have completed bachelor degree or less.

Most of the nurses have agreed on measures to prevent VAP such as staff education, protocolized approach, creation of structures to facilitate bundle adherence and regular feedback to the system. They scored an average of $60.5 \%$ in this front. This finding suggests improving the nursing education system and including basic approach for disease prevention in the nursing program. ${ }^{12}$

Hand hygiene is a part of universal precaution and every infection prevention bundle as it helps to prevent infection from transient organisms. Centre for Disease Control in the United States strongly recommends hand washing and failure to do so between patients is associated with an increased incidence of VAP. ${ }^{13}$ The knowledge on hand hygiene was $94.2 \%$. Since hand hygiene is mandatory for critical care areas in this centre, it can be inferred that the practice of hand hygiene has been translated to their knowledge.

All the respondents knew that a close in-line suction system prevents VAP. This finding is congruent to Heyland ${ }^{14}$ study which reveals that closed suction systems are used in $88 \%$ of ICUs. Nurses in this study find close in-line suction relatively convenient, as it can be simply executed with clean gloves without seeking other's help and avoid the sterile technique and lengthy procedure of open suction.

Meanwhile, semi-recumbent positioning to (3045) degrees was well acknowledged by nurses to prevent VAP (score $88.6 \%$ ). Because this position is easier and convenient for patients, nurses also seem to favor this. Overall knowledge of the nurses on this aspect was found to be quite good (86\%), which is very similar to the study done by PérezGranda $^{15}$ and El-Khatib ${ }^{16}$ where $92.6 \%$ and $97 \%$ of respondent nurses respectively consider this position to prevent VAP.

Regarding ventilation and intubation techniques, the average score was $72.8 \%$ and approximately $27(47 \%)$ nurses preferred non-invasive ventilation while 30 (52.6\%) preferred oral intubation to prevent VAP. This finding is similar to El-Khatib ${ }^{16}$ 
and Korhan $\mathrm{EA}^{17}$ where $76 \%$ and $79 \%$ of the nurses respectively knew that oral intubation is recommended for reducing the risk of VAP. Also, it was found that 30 (52.6\%) nurses viewed on the requirement to change ventilator circuit for each patient which is similar to the study done by Korhan, which revealed $62.3 \%$ for the same. ${ }^{17}$ This centre has been practicing oro-gastric tube feeding for the mechanically ventilated patients. Hence, the average score computed was 98.8\% with $56(98.2 \%)$ nurses favoring oro-gastric tube to decrease the overall incidence of VAP.

The knowledge score on kinetic bed was 36.8\%. Approximately 51 (89.5\%) nurses believe that kinetic bed decreases the risk of VAP while in actual it does not. The study done by Korhan ${ }^{17}$ and Labeau $^{18}$ has showed $54.3 \%$ and $57.3 \%$ of nurses respectively believed that kinetic bed decreases the risk of VAP. The unavailability and unawareness of this expensive beds might have contributed to this wrong perception.

Approximately 56 (98\%) respondents knew that the ETT with an extra lumen for sub-glottic suction prevents VAP which is very similar to the study conducted by El-Khatib ${ }^{16}$ where $97 \%$ of nurses knew it. In contrast to this study, Labeau18 and Korhan $^{17}$ found $50.6 \%$ and $23.9 \%$ of nurses respectively knew that ETT with sub-glottic suction prevents VAP. The knowledge score of the nurses in this study is better which can be attributed to the hospital practice where ETT with sub-glottic suction is readily available, frequently used and familiar with the intubating physicians.

In this study, knowledge score on change of humidifier was $64.5 \%$, and 16 (28.1\%) respondent knew that humidifier needs to be changed every week or when clinically indicated. This is very similar to the study conducted by El-Khatib ${ }^{16}$ where it was known to $26 \%$ of nurses.

The respondents had least average knowledge score of $36.3 \%$ about the sedation holiday. Approximately, $46(80 \%)$ respondents were unaware that sedation holiday needs to be continued until the patient is fully awake and comfortable or deemed to resumtion of sedation due to agitation, while none knew that sedation holiday prevents VAP. SBT has a low score $(46.2 \%)$ in the VAP prevention strategy. For those patients receiving mechanical ventilation, sedation holiday decreases ventilation duration. ${ }^{19}$ As the risk of VAP is related to the duration of mechanical ventilation, $^{20}$ limiting this duration via sedation holiday reduces the VAP. In this study, almost half of the respondents knew that early mobility is useful to prevent VAP.

The knowledge score was $66.7 \%$ for oral and digestive tract care to prevent VAP with 56 (98.2\%) nurses favoring the use of oral chlorhexidine mouth wash to prevent VAP. This practice is common and widely used by nurses, and favored by several systemic reviews and comparative study. ${ }^{21,22}$ Selective digestive decontamination (SDD) showed a significant reduction in mortality and morbidity with the effect of resistance being controlled or unknown.23 SDD is not widely practiced in this centre, mainly due to insufficient evidence, concern about resistance and that SDD has recently been ranked the worst maneuver for preventing VAP by an expert panel. ${ }^{24}$ Only 5.3\% of nurses favored SDD as a measure to prevent VAP in this study.

The fact that regular measurement of cuff pressure is a good technique to prevent micro-aspiration and VAP is known to 56 (98.5\%) nurses. A study by Fernandez ${ }^{25}$ showed that the ETT with ultrathin polyurethane(PU) cuff to prevent VAP is inconsistent without bundles of care and this tube is relatively expensive and unavailable in all the settings. Also, Sashes ${ }^{26}$ found no statistically significant reduction in the incidence of VAP when they compared PU cuffed ETT to conventional ETT. Only 10 (17.5\%) nurses in this study thought such a PU ETT can prevent VAP. Being tertiary centre, late presentation of patient is common and to avoid the risk of surgical site of infection post-surgery, prescription of antibiotics is always favoured. This tendency seems to have an impact on knowledge among nurses and so their average knowledge score for antibiotics and probiotics is $41.5 \%$ and only 10 (17.5 $\%$ ) of nurses favor probiotics to prevent VAP.

Association was not significant between knowledge score on prevention of VAP and years of experience in ICU ( $p<0.05)$, which is similar to the study done in South Africa where there was no association between the level of education, ICU training, years of experience and knowledge on prevention of VAP. $^{27}$

Though the findings of this study are congruent with literature, it has several limitations. This study was conducted in a single centre and even though nurses working in various critical care areas were enrolled, the sample size is small. As the study is conducted in a cardiac centre, its findings may not be extrapolated to other multidisciplinary centres. Since the qualitative data was not collected, validation, further analysis, and triangulation of quantitative data with the qualitative information from the center's management team was not possible. However, the lead author, who works in the same center has insights and understanding about hospital management and some of the imperatives discussed in the paper are based on his personal experiences and observation.

\section{CONCLUSION}

In conclusion, the nurses working in critical care areas in a tertiary cardiac centre of Nepal have overall good knowledge on the prevention of VAP. 
But, they have poor theoretical knowledge of the protocolized approach and definition of VAP. In light of these, this study recommends to incorporate more nurses in training related to infection and VAP prevention, both in-house and outside.

\section{CONFLICT OF INTEREST}

None declared.

\section{REFERENCES}

1. Milt $C$, Pincirolhi $R$, Patel $N$ and Berra L. Ventilator associated Pneumonia: evolving definition and preventive strategies. Respir Case. 2013:58:990-1007.

2. Klompas M, Branson R, Eichenwald EC, Greene LR, Howell MD, Lee $G$, et al. Strategies to Prevent Ventilator-Associated Pneumonia in Acute Care Hospitals 2014 Update. Infect Control Hosp Epidemiol. 2014:35:915-36.

3. Hayashi Y, Morisawa K, Klompas M, Jones M, Bandeshe H, Boots $R$, et al. Toward improved surveillance: the impact of ventilatorassociated complications on length of stay and antibiotic use in patients in intensive care units. Clin Infect Dis. 2013;56:471-7.

4. Safdar N, Dezfulian C, Collard HR and Saint S. Clinical and economic consequences of ventilator-associated pneumonia: a systematic review. Crit Care Med 2005;33:2184-93.

5. Novosel TJ, Hodge LA, Weireter L, Britt RC, Collins JN, Reed SF, et al. Ventilator-associated pneumonia: depends on your definition. Am Surg. 2012;78:851-54.

6. Thomas BW, Maxwell RA, Dart BW, Hartmann EH, Bates DL, Mejia VA, et al. Errors in administrative-reported ventilator associated pneumonia rates: are never events really so? Am Surg. 2011;77:998-1002.

7. Burja S, Belec T, Bizjak N, Mori J, Markota A, Sinkovič A. Efficacy of a bundle approach in preventing the incidence of ventilator associated pneumonia (VAP). Bosn J Basic Med Sci. 2018;18(1):105109. doi:10.17305/bjbms.2017.2278

8. O'Meara ES, White M, Siscovick DS, Lyles MF and Kuller LH. Hospitalization for Pneumonia in the Cardiovascular Health Study: Incidence, Mortality, and Influence on Longer-Term Survival. J Am Geriatr Soc. 2005;53:1108-16.

9. Myrianthefs, PM, Kalafati M, Samara I and Baltopoulos GJ. Nosocomial pneumonia. Crit Care Nurs Q. 2004;27:241-57.

10. Babcock HM, Zack JE, Garrison T, Trovillion E, Jones M, Fraser VJ, et al. An educational intervention to reduce Ventilator associated pneumonia in an integrated health system: a comparison of effects. Chest. 2004;125: 2224-3.

11. Ali NS. Critical Care Nurses' Knowledge and Compliance with Ventilator Associated Pneumonia Bundle at Cairo University Hospitals. J Edu Pract; 2013;4:66-77.
12. Ruffle $A$ and Adamcova L. Ventilator-associated Pneumonia: Prevention Is Better Than Cure. Nuri crit Care. 2008;13:44-53.

13. Wu D, Wu C, Zhang $S$ and Zhong Y. Risk Factors of VentilatorAssociated Pneumonia in Critically III Patients. Front Pharmacol. 2019;10:482.

14. Heyland DK, Cook DJ and Dodek PM. Prevention of ventilator associated pneumonia: current practice in Canadian critical care units. J Crit Care. 2002;17:161-7.

15. Perez-Granda MJ, Munoz P, Heras C, Sanchez G, Rello J, Bouza E, et al. Prevention of Ventilator-Associated Pneumonia: Can Knowledge and Clinical Practice Be Simply Assessed in a Large Institution? Respir Care 2013;58:1213-9.

16. El-Khatib MF, Zeineldine S, Ayub C, Husari A and Bou-Khalil PK. Critical care clinicians' knowledge of evidence based guidelines for preventing Ventilator associated pneumonia. Am J Crit Care. 2010;19:272-6.

17. Korhan EA, Yont GH, Kilic SP and Uzelli D. Knowledge levels of intensive care nurses on prevention of ventilator-associated pneumonia. Nurs Crit Care. 2014;19:26-33.

18. Labeau S, Vandijck D, Rello J, Adam S, Rosa A, Wench C, et al. Evidence-based guidelines for the prevention of ventilatorassociated pneumonia: results of a knowledge test among European intensive care nurses. J Hosp Infect 2008;70:180-5.

19. Kress JP, Pohlman AS, O' Connor MF and Hall JB. Daily interruption of sedative infusions in critically ill patients undergoing mechanical ventilation. N Engl J Med. 2000;342:1471-7.

20. Cook DJ, Walter SD, Cook RJ, Griffith LE, Guyatt GH, Leasa D, et al. Incidence of and risk factors for ventilator-associated pneumonia in critically ill patients. Ann Intern Med. 1998; 129:433-40.

21. Snyders 0 , Khondowe 0 and Bell J. Oral chlorhexidine in the prevention of ventilator-associated pneumonia in critically ill adults in the ICU: A systematic review. SAJCC 2011;27: 48-52.

22. Koeman M, van der Ven AJM, Hak E, Joore HCA, Kaasjager K, Smet $A G A$, et al. Oral decontamination with chlorhexidine reduces the incidence of ventilator-associated pneumonia. Am J Respir Crit Care Med 2006;173:1348-55.

23. Rello J, Lode H, Cornelia G, Masterson R and VAP care Bundle Contributors. European care bundle for prevention of Ventilator associated pneumonia. Intensive care Med. 2010;36:733-80.

24. Vincent JL. Selective digestive decontamination for everyone, everywhere? Lancet 2003; 362:1006-7.

25. Fernandez JF, Levine SM, Restrepo MI. Technologic advances in ETT for prevention of Ventilator associated Pneumonia. Chest 2012;142:231-8

26. Sashes $P$, Kundra $P$ and Cherian A. Polyurethane cuffed Vs Conventional Endotracheal Tube: effect on VAP rates and length of ICU stay. Indian J. Anaesth. 2016;60:163-7.

27. Labeau S, Vandijck D, Aken PV, Claes B, Blot S. Intensive care nurses' knowledge of evidence-based guidelines for the prevention of ventilator-associated pneumonia. Crit Care. 2007;52:92. 